\title{
Simulations of Ly $\alpha$ Absorption from Low Surface Brightness Galaxies
}

\author{
Suzanne M. Linder \\ Pennsylvania State University, University Park, PA 16802 USA; \\ slinder@astro.psu.edu
}

\begin{abstract}
Using simulations of the low redshift galaxy population based upon galaxy observations, it is shown (Linder 1998) that the majority of Ly $\alpha$ absorbers at low redshift could arise in low surface brightness (LSB) galaxies. The contribution to absorption from LSB galaxies is large for any galaxy surface brightness distribution which is currently supported by observations. Ly $\alpha$ absorbers should become powerful tools for studying the properties and evolution of galaxies, but first it will be necessary to establish observationally the nature of the Ly $\alpha$ absorbers at low redshift. Further simulations, in which the absorbing galaxy population is 'observed' with some selection criteria, are used to explore how easy it is for an observer to test for a scenario in which LSB galaxies give rise to most of the Ly $\alpha$ absorbers. It is shown that absorption arising in LSB galaxies is often likely to be attributed to high surface brightness galaxies at larger impact parameters from the quasar line of sight.
\end{abstract}

\section{Introduction}

Ly $\alpha$ absorption at low redshift is a powerful probe of gas in or around galaxies. With Ly $\alpha$ absorber observations it is possible to detect neutral hydrogen column densities which are several orders of magnitude lower than those typically seen with $21 \mathrm{~cm}$ observations. For example, the HST Key Absorption Line project (Bahcall et al. 1996) is complete to approximately $N_{H I} \sim 10^{14.3}$ $\mathrm{cm}^{-2}$. Observations of Ly $\alpha$ absorption should therefore be capable of detecting neutral gas which is known to exist in galaxy disks as well as more diffuse gas or neutral components of highly ionized gas surrounding galaxies. Given that Ly $\alpha$ absorption lines can be observed even more easily at higher redshifts, they should become especially useful tools for studying galaxy evolution in the future.

Some stronger Ly $\alpha$ absorption lines are known to arise in lines of sight thorough galaxies, but the nature of the more common, weak forest absorbers is more difficult to establish. Supposing that these absorbers arise in gas associated with galaxies, the galaxies may be located at large impact parameters from a quasar line of sight. Therefore it becomes impossible to be sure that any particular galaxy is actually causing the absorption, so that it is difficult to make a direct test of any model in which galaxies cause absorption.

Testing absorber-galaxy models becomes even more complicated if absorption arises in unidentified galaxies. Given that low surface brightness (LSB) 


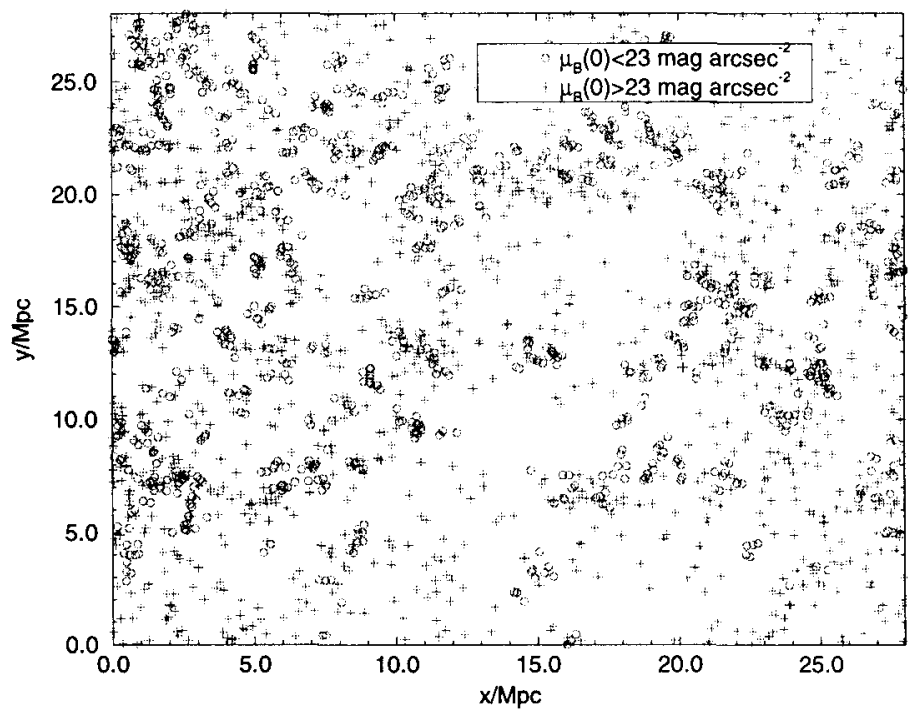

Figure 1. A slice of the cluster simulation which is $5 \mathrm{Mpc}$ thick is shown, illustrating the positions of LSB and HSB galaxies. LSB galaxies are made to be clustered more weakly than HSB galaxies.

galaxies are common relative to high surface brightness (HSB) galaxies, and that LSB galaxies are often found to be rich in gas, they must make an important contribution to Ly $\alpha$ absorption. LSB galaxies are typically bigger than HSB galaxies at a given luminosity. If LSB galaxies also have larger sizes compared to HSB galaxies in Ly $\alpha$ absorption, then they may easily dominate the cross section for Ly $\alpha$ absorption. Ly $\alpha$ absorbers may allow for an important way to study the properties and evolution of galaxies, especially LSB galaxies, but first it will be necessary to find a way to test absorber-galaxy models and to establish what types of galaxies could actually cause most of the Ly $\alpha$ absorption.

\section{Simulations of Absorbing Galaxies}

Simulations are used (Linder 1998) to find out how many absorbers could be associated with galaxies. A population of galaxies is defined according to observed distributions of galaxy parameters, and the galaxies are placed in a box. Random lines of sight are chosen to go through the box, and absorption occurs where the lines of sight intersect the galaxies. In these simulations it is assumed that absorption arises in galaxy disks and their ionized outer extensions (Charlton, Salpeter \& Linder 1994). It is shown that Ly $\alpha$ absorber counts at low redshift can easily be explained by galaxies when LSB galaxies are included. The model parameters are tuned to be consistent with observed luminosity functions for HSB and LSB galaxies, standard nucleosynthesis predictions of the baryon 


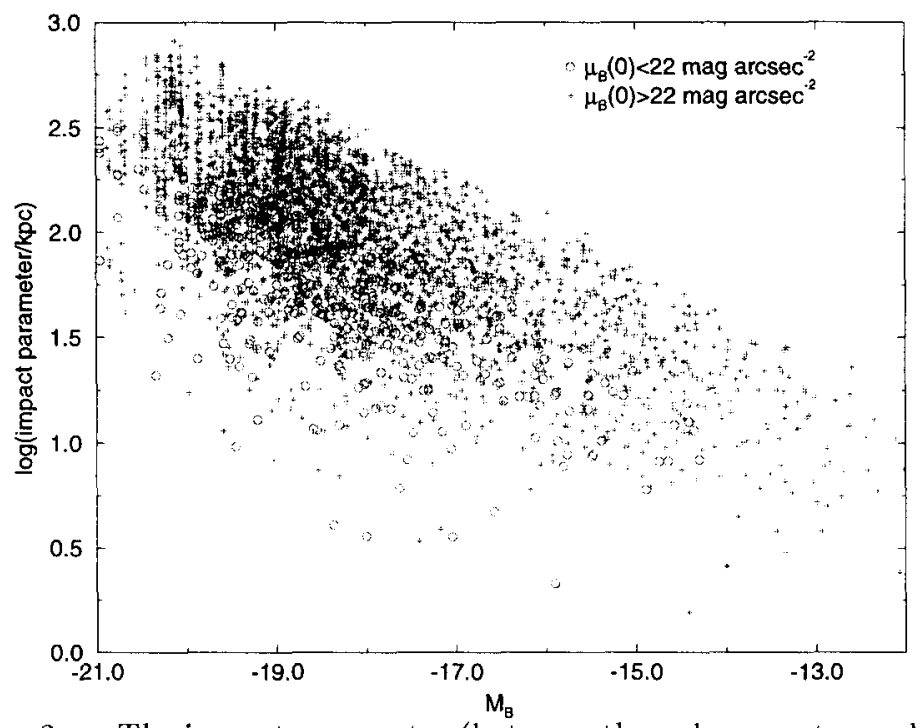

Figure 2. The impact parameter (between the galaxy center and the quasar line of sight) is plotted versus $M_{B}$ for the actual absorbing $\left(>10^{14.3} \mathrm{~cm}^{-2}\right.$ ) galaxies in the simulations. More luminous galaxies have larger absorption radii, and can thus cause absorption at larger impact parameters from the line of sight. LSB galaxies have larger absorption radii than HSB galaxies at a given luminosity.

density, and Lyman limit absorber counts. Preferred scenarios are found to have moderate galaxy absorption cross sections and moderate numbers of LSB galaxies.

The galaxies in the earlier simulations are placed randomly in the box. More recently, clustered galaxies have been simulated using a fractal-type method as in Soniera \& Peebles (1978) where eight levels of clustering are used. The LSB galaxies are moved further out from the centers of the clusters at the second largest level, so that they are made to be clustered more weakly than HSB galaxies, as shown in a slice of the box in Fig. 1.

More luminous galaxies have larger absorption radii in the simulations, so that they are able to cause absorption when they are at larger impact parameters from quasar lines of sight, as shown in Fig. 2. The Holmberg relation $(R \sim$ $L^{0.5}$ ) and the typical absorbing radius of a luminous HSB galaxy are similar to those reported in some observational studies (Chen et al. 1998; Lanzetta, this Proceedings). It can also be seen from Fig. 2 that LSB galaxies have larger absorption radii than HSB galaxies at a given galaxy luminosity. Thus the majority of Ly $\alpha$ absorbers arise in lines of sight through LSB galaxies, as seen in Fig. 3. It can also be seen in Fig. 3 that the Ly $\alpha$ absorption is generally 


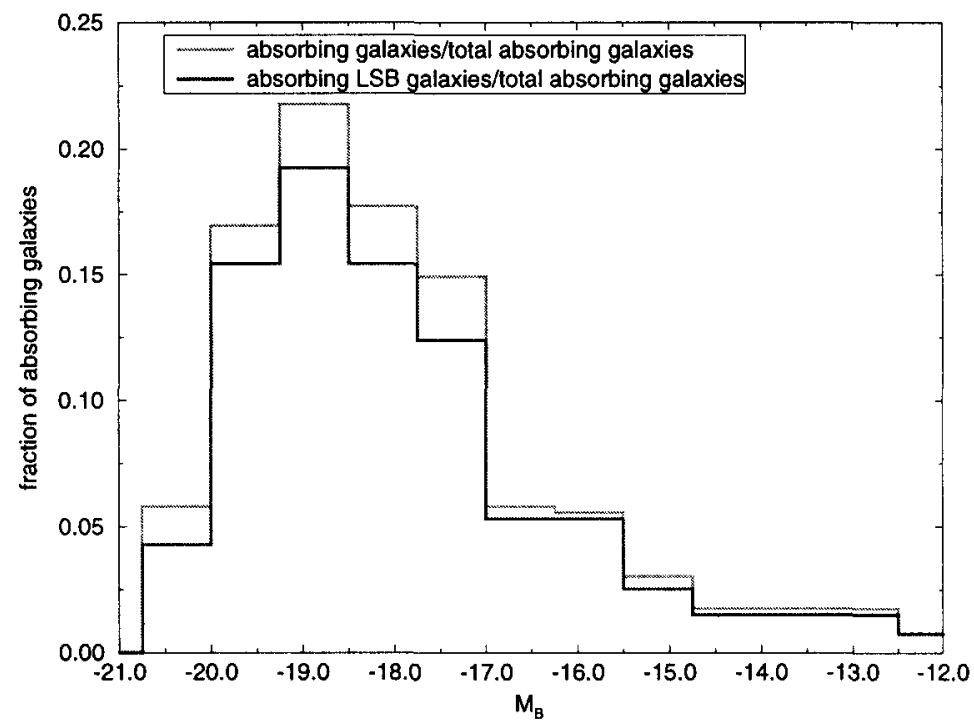

Figure 3. A distribution of $M_{B}$ is shown for the actual absorbing $\left(>10^{15} \mathrm{~cm}^{-2}\right.$ ) LSB (> $22 \mathrm{~B} \mathrm{mag} \operatorname{arcsec}^{-2}$ ) galaxies and all galaxies, both normalized to the total number of absorbing galaxies. Moderately luminous LSB galaxies are shown to cause most of the Ly $\alpha$ absorption.

caused by moderately luminous, 'normal' LSB galaxies and not by extremely luminous Malin-type objects.

A flat central surface brightness distribution (McGaugh 1996) at a given scale length was assumed for most of the simulations. Preliminary simulations have also been done using a surface brightness distribution which is lognormal at a given luminosity (de Jong, this Proceedings). While this surface brightness distribution allows for fewer extremely luminous LSB galaxies, there are still numerous moderately luminous LSB galaxies which still give rise to most of the Ly $\alpha$ absorption.

\section{3. 'Observing' the Simulations}

Given some scenario where LSB galaxies make a significant contribution to Ly $\alpha$ absorption, such as the one described above, is it possible for an observer to test for such a scenario? Suppose we can 'observe' the galaxies simulated above using some selection criteria. Here the nearest galaxy to a line of sight is found within a velocity difference of $400 \mathrm{~km} \mathrm{~s}^{-1}$ to the absorption line, where the galaxy has $M_{B}<-16$ and $\mu_{B}(0)<23 \mathrm{mag} \operatorname{arcsec}^{-2}$.

A plot of $M_{B}$ versus the impact parameter of the 'observed' absorining galaxy is shown in Fig. 4, using the same scale as the actual plot shown in Fig. 2. It is likely that an observer could find a way of determining that the 


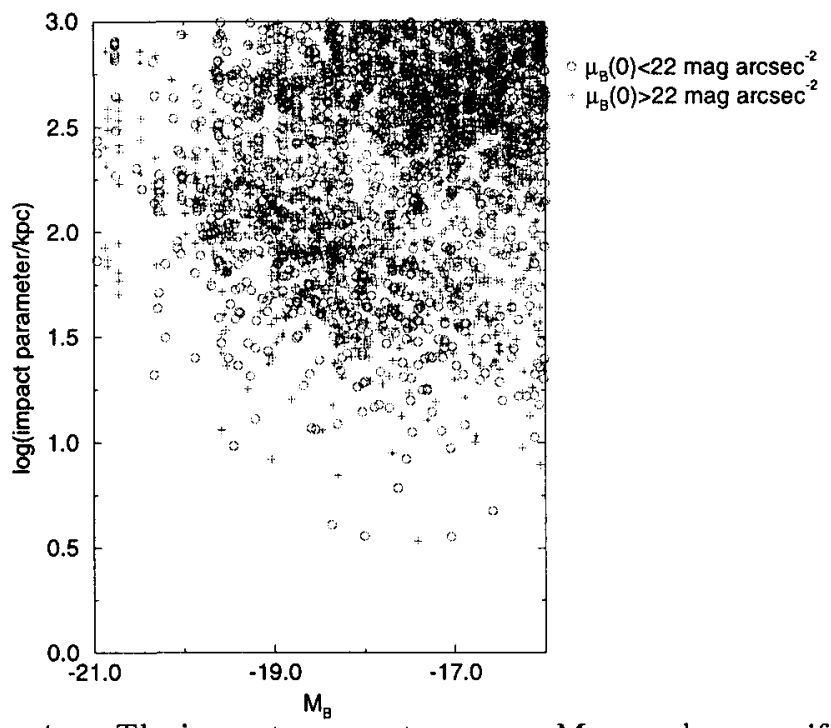

Figure 4. The impact parameter versus $M_{B}$ are shown as if they were obtained by 'observing' the simulated galaxies, in order to identify an absorbing galaxy, according to the selection criteria given in the text. While the same simulated galaxies shown in Fig. 2 are 'observed' here, it is no longer obvious that LSB galaxies have larger absorption cross sections.

points in the upper right-hand corner for Fig. 4 are unphysical absorber-galaxy associations. For the remaining points, a correlation between luminosity and impact parameter can still be seen, although the slope of the 'observed' Holmberg relation may change. However, absorption arising in LSB galaxies is frequently 'observed' as arising in HSB galaxies at typically larger impact parameters from the quasar line of sight. Thus it is no longer possible to verify from the 'observed' plot that LSB galaxies have larger absorption cross sections, as assumed in the simulation.

In any reasonable absorber-galaxy model, it is expected that, on average, the neutral hydrogen column density should fall off with galaxy radius. Thus it is likely that absorbing galaxies at larger impact parameters from the quasar line of sight should produce lower column densities. An anticorrelation between impact parameter and neutral column density is seen for the simulated actual absorbing galaxies (Linder 1998). However, a large amount of scatter exists in such plots, which is caused mostly by variations in the properties of absorbing galaxies. (Varying disk inclinations produce comparatively little scatter, so that a spherical halo-type model for absorbing galaxies should produce a similar plot.)

The anticorrelation between neutral column density and impact parameter can still be seen for the galaxies 'observed' with the selection criteria above, as shown in Fig. 5. Note that if more reasonable selection effects were used to 


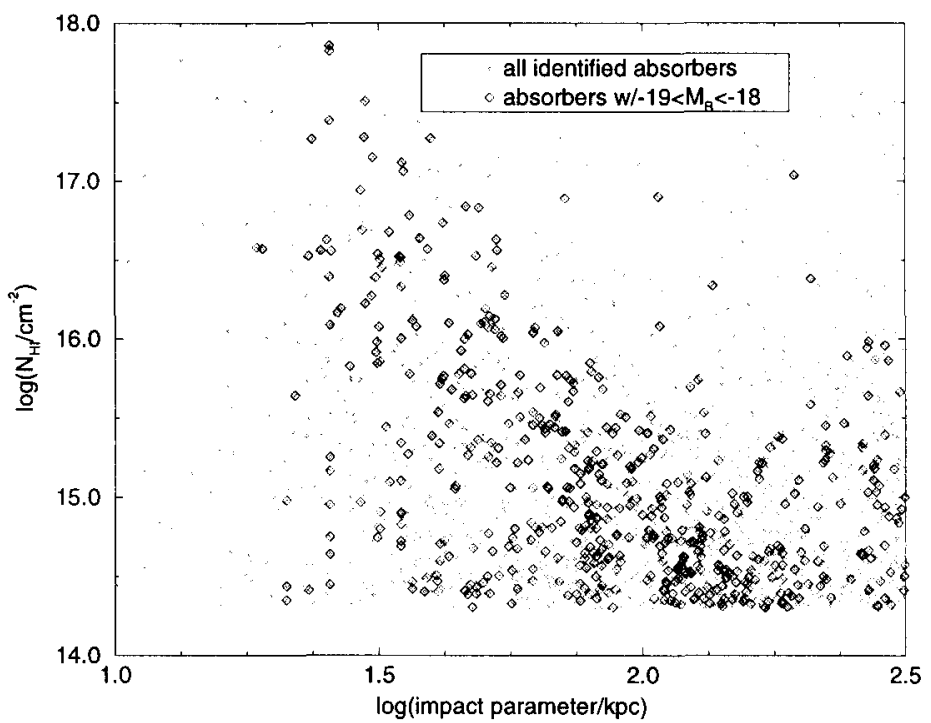

Figure 5. The neutral column density for Ly $\alpha$ absorption is plotted versus the impact parameter between galaxy and line of sight for the 'observed' (see text) absorbing galaxy. An anticorrelation between neutral column density and impact parameter is still seen even though the actual absorbing galaxy is unlikely to be identified (particularly for the weaker absorption lines).

'observe' the simulations, compact HSB dwarf galaxies would be excluded, so that fewer points would be seen toward the lower left-hand corner. Thus, when also considering incompleteness for observing galaxies at large impact parameters, Fig. 5 should bear even more resemblance to the plot shown in Chen et al. (1998). LSB absorbers are frequently misidentified in the simulation shown. In fact, many LSB absorbers have no possible 'observed' galaxy which satisfies the selection criteria, especially when the LSB galaxies are clustered more weakly than the HSB galaxies. Thus an observer would be likely to conclude that some fraction of absorbers cannot be explained by galaxies. Less scatter is seen in Fig. 5 for galaxies with a narrow range of luminosities. However, it is more difficult to reduce the scatter by limiting the range of 'observed' absorbing galaxy surface brightnesses. Thus from this plot an observer is likely to conclude again that galaxy absorption cross sections are correlated with galaxy luminosity but not with galaxy surface brightness.

Another plot frequently made by observers (for example, Bowen, Blades, \& Pettini 1996), that illustrates the absorption covering factor, or the fraction of galaxies found to cause absorption as a function of impact parameter, is shown in Fig. 6. 'Observed' galaxies appear to cause absorption at large impact parameters compared to those seen in Fig. 2. Again it can be seen that many 


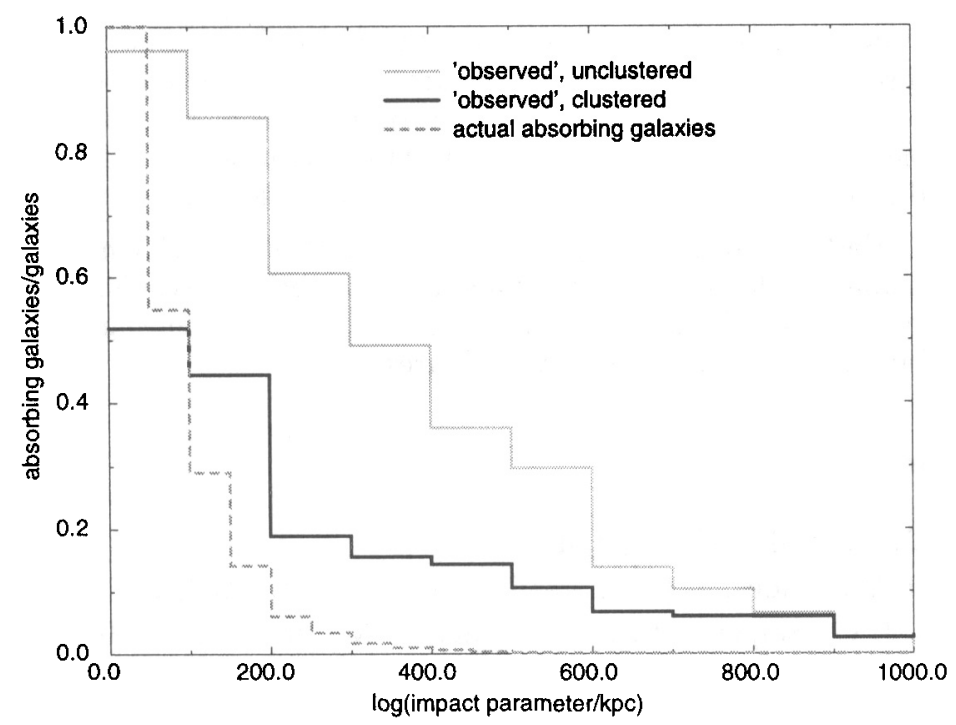

Figure 6. A 'covering factor' plot shows the fraction of galaxies found at some impact parameter from a line of sight which cause absorption $\left(>10^{14.3} \mathrm{~cm}^{-2}\right)$. Galaxy absorption radii are likely to be overestimated when the galaxies are observed with strong selection effects against LSB galaxies. Galaxy clustering also causes misidentification of the actual absorbing galaxy to occur more frequently.

absorbers arising in LSB galaxies are attributed to HSB galaxies at larger impact parameters from the quasar line of sight. It can also be seen that clustered galaxies are more frequently misidentified. It will be difficult to use an observed covering factor plot to test an absorber-galaxy model since its appearance will be sensitive to the actual absorbing properties of the galaxies, the clustering behavior of the galaxies, and the observational selection criteria.

\section{Conclusions}

It has been shown that $\operatorname{Ly} \alpha$ absorbers at low redshift can easily be explained by galaxies when LSB galaxies are included. The majority of absorbers are likely to arise in LSB galaxies, even if extremely luminous LSB galaxies are rare. Ly $\alpha$ absorber observations will be useful for constraining properties of galaxies, such as the gaseous extent of galaxies, and for studying evolution in such galaxy properties. Ly $\alpha$ absorbers will be especially powerful tools for studying galaxies which are LSB at any evolutionary stage.

In order to take advantage of the information from Ly $\alpha$ absorption, it will be necessary to establish observationally specifically what kinds of galaxies give rise to Ly $\alpha$ absorption. Observational studies which attempt to match absorption 
lines with possible absorbing galaxies will be crucial to establishing the nature of the Ly $\alpha$ absorbers. However, as shown above, it is also necessary to use simulations in order to understand the absorbing properties of galaxies. For example, an observer may overestimate the absorbing radius of a galaxy by looking directly at observations which are subject to severe selection effects. Selection effects will always be present in observational studies, but it should be possible to define the selection criteria more rigorously so that they can be simulated in order to make more realistic tests of absorber-galaxy models.

Acknowledgments. I am grateful to J. Charlton and C. Churchill for helpful discussions, R. de Jong for supplying a surface brightness distribution before publication, WISE CIC and NASA GSRP for travel funding, and R. Gunesch and A. Panaitescu for assistance with figures.

\section{References}

Bahcall, J. N. et al. 1996, ApJ, 457, 19

Bowen, D. V., Blades, J. C., \& Pettini, M. 1996, ApJ, 464, 141

Charlton, J. C., Salpeter, E. E., \& Linder, S. M. 1994, ApJ, 430, L29

Chen, H, -W., Lanzetta, K. M., Webb, J. K., Barcons, X. 1998, ApJ, 498, 77

Linder, S. M. 1998, ApJ, 495, 637

McGaugh, S. S. 1996, MNRAS, 280, 337

Soniera, R. M. \& Peebles P. J. E. 1978, AJ, 83, 845 